Sharif University of Technology
Scientia Iranica

\title{
Investigation of harmonic effects in locational marginal pricing and development of a framework for LMP calculation
}

\author{
M. Narimani and S.H. Hosseinian* \\ Department of Electrical Engineering, Amirkabir University of Technology, Tehran, Iran. \\ Received 23 September 2019; received in revised form 7 December 2019; accepted 8 February 2020
}

\section{KEYWORDS}

Locational marginal pricing;

Harmonic;

Optimal power flow;

Nodal price;

Skin effect.

\begin{abstract}
Locational Marginal Pricing (LMP) is arguably the most effective and commonly employed mechanism to provide the most reliable economic signal for market participants. Meanwhile, nodal prices depend on active power losses and transmission congestion which may be affected by harmonics pollution. In the conventional method, power system and loads are assumed linear and nodal prices are obtained by results of Optimal Power Flow (OPF) at the power frequency. Harmonics lead to skin effect and greater loss. Further, harmonic flowing in branches in a power network occupies transmission capacity. For providing more accurate signals to market participants and achieving more accurate nodal prices, harmonic effects on LMP are investigated and a framework is developed for LMP calculation in a harmonic polluted power system. In this framework, skin effect, losses, and congestion that can be arisen from harmonic pollution are modeled in OPF and are considered in LMP calculation. The proposed concept is implemented with 9-bus and 30-bus test systems, while nodal price changes are also indicated.
\end{abstract}

(C) 2022 Sharif University of Technology. All rights reserved.

\section{Introduction}

Locational Marginal Pricing (LMP) is one of the most commonly used tools employed in the electricity market to determine nodal prices and provide economic signals for market participants. LMP is defined as the cost of supplying the next load increment at that bus. Many of the well-known running power markets such as PJM, NYISO, ISO-NE, CAISO, ERCOT, MISO, and NEMCO have utilized LMP in their systems $[1,2]$.

\footnotetext{
*. Corresponding author. Tel.: +98216454 3343; Fax: +982164543301

E-mail address: hosseinain@aut.ac.ir (S.H. Hosseinian)
}

Optimal Power Flow (OPF) results are sensitive to the constraints of the branch flow and the way the transmission losses are spotted in calculations [3].

Accurate values of each component of the price are required to achieve the desired purpose of LMP signals, i.e., efficient dispatch and fruitful incentives. Accordingly, consideration of the variations in the system parameters for assessing changes in LMPs is beneficial to the bidding strategies of producers and consumers [4].

A market framework for the practical implementation of lossy Financial Transmission Rights (FTRs) was proposed in [5]. The lossy FTRs can be settled directly according to LMPs without requiring any LMP decomposition.

The lossy FTR mechanism was improved and 
upgraded through the introduction of lossy option FTRs in [6].

In [7], a mathematical model was provided for adding cost components of loss and power factor to the transmission pricing.

In [8], current pricing was proposed as a method to achieve nodal prices without marginal losses for DC grids. The nodal prices without marginal losses were derived by linearizing the quadratic OPF problem only through fixing the voltage, instead of using Taylor approximation.

Authors in [9] discussed the pricing of marginal transmission network losses in the LMP. In this paper, the traditional loss model was studied and a new model was proposed. The proposed model achieved more defendable and predictable market-clearing results by introducing loss distribution factors to explicitly balance the consumed losses in the lossless DC power system model.

In [4], a new model was presented to evaluate harmonic losses in OPF and nodal pricing. The presence of harmonic losses in the OPF problem was expressed as particular resistive elements added to the line impedance in this model. Also, the reactance variations were assumed to be a multiplication of the harmonic order. Skin effect and resistance changes were not considered in the referenced study.

A new LMP policy for the distribution system was presented in [10] with significant penetration of Distributed Generation (DG). Despite acknowledging that significant losses play an essential role in the LMP calculation, the effect of losses due to network imbalances and harmonic was considered.

The dynamic tariff concept was introduced in [11] and based on the Distribution Locational Marginal Pricing (DLMP) for solving congestion problems, obtained by controlling the price values at different nodes. This analysis was extended in [12] to consider intertemporal characteristics of the flexible load. A new quadratic programming-based approach was presented in [13] to address the multiple solutions of the decentralized aggregator optimization algorithm utilized in [12].

A simple methodology was described in [14] based on the analysis performed by the Hydro One in-house Computer Program (PROCOSE) to calculate Transmission Congestion Cost (TCC) for a given period of time in dollars per unit time and LMP in dollars per Megawatt-hour (MWh) at any selected bus in the transmission system. Sensitivity studies for determining the effect of changes in the system parameters and operating conditions on the LMPs can be carried out using this methodology.

A congestion management approach taking the demand elasticity into account was proposed in [15]. Meanwhile, the issue of harmonic losses is always dis- puted in a deregulated power system. The rapid development of the industries and energy supply technology improvements are the main contributing factors of the harmonic distortion [16]. Many industrial consumers employing electronic devices such as reactive power compensation devices and UPSs are the significant factors of harmonic distortion in the distribution network [17]. For example, Korea will develop the infrastructure battery charging for electric vehicles that can increase harmonic pollution [18]. Utility companies usually install mitigation equipment to maintain the quality of power supply. The cost of this installed equipment must be recovered from the consumers who have caused the power quality problem, i.e., harmonic distortion [19].

In [20], a market-based framework was proposed for central management of harmonic compensation actions in Micro Grids (MGs). To this end, a distortion power expected payment function (DEFP) was proposed for each Active Power Filter (APF) representing various imposed costs for participation in the Harmonic Power Market (HPM).

With the development of power electronics technology, nonlinear loads acting as harmonic current sources are increasing in power distribution systems [21-25]. Harmonics cause reactance variations, skin effect, heightened resistance of lines, and increased line losses. Further, harmonics occupy the capacity of the lines, which can cause changes in the power market signals to inaccurate signals. Thus, these effects should be considered in pricing, which were remained neglected in the above-mentioned papers. In [4], only harmonic loss and reactance variations were considered in LMP, but skin effect and capacity occupation were not included. The main focus of our work is incorporate these effects in nodal pricing. To this end, these items are modeled in a way that can be used in pricing without altering the basic OPF equations.

The outline of this paper is as follows: In Section 2 , the required formulations for LMP, OPF, and skin effect are presented. The proposed algorithm for nodal price calculation by considering harmonic is discussed in Section 3, which is followed by two case studies in Section 4. Section 5 concludes the paper.

\section{Formulation}

\subsection{Locational Marginal Pricing (LMP)}

The theoretical price of electricity at each node on the network is a calculated shadow price; it is assumed that the next increment of electric energy at a specific bus and the hypothetical incremental cost to the system that would result from the optimized dispatch of available units establish the hypothetical production cost of the hypothetical incremental demand.

LMP is the summation of the marginal cost 
of generation, congestion cost, and cost of marginal losses [26]:

$$
\lambda_{n}=\lambda_{\text {ref }}+\lambda_{\text {cong }}+\lambda_{\text {loss }} .
$$

Thus, LMP is a function of line congestion and losses. As mentioned above, intensified losses and line capacity occupation are the effects of harmonics.

The LMPs can be computed by either AC Optimal Power Flow (ACOPF) or DC Optimal Power Flow (DCOPF). The ACOPF model is more accurate than the DCOPF model, but is prone to divergence. Although the ACOPF form is complex, it has been implemented by NYISO (New York International System Organization) and CAISO (California Independent System Operator) [27-29].

\subsection{Optimal Power Flow (OPF)}

The combination of an objective function and the power flow equations results in OPF. Most OPF variants are based on the classical formulation of Carpentie [30] and Dommel and Tinney [31]. The classical formulation is an extension of the classic economic dispatch. The economic dispatch problem can be thought of as maximizing the economic welfare of a power network or minimizing the total cost of electricity generation while meeting the system constraints.

The ultimate purpose is to optimize the total cost of generation, with the classical form of the formulation being as follows:

$$
\min \sum_{n \in \mathbf{G}} C_{n}\left(P_{n}^{G}\right)
$$

s.t.:

$$
\begin{aligned}
& P_{n}(V, \delta)=P_{n}^{G}-P_{n}^{L} \quad \forall n \in \mathbf{N}, \\
& Q_{n}(V, \delta)=Q_{n}^{G}-Q_{n}^{L} \quad \forall n \in \mathbf{N}, \\
& P_{n}^{G, \text { min }} \leq P_{n}^{G} \leq P_{n}^{G, \text { max }} \quad \forall n \in \mathbf{G}, \\
& Q_{n}^{G, \text { min }} \leq Q_{n}^{G} \leq Q_{n}^{G, \text { max }} \quad \forall n \in \mathbf{G}, \\
& V_{n}^{\min } \leq V_{n} \leq V_{n}^{\max } \quad \forall n \in \mathbf{N}, \\
& \delta_{n}^{\min } \leq \delta_{n} \leq \delta_{n}^{\max } \quad \forall n \in \mathbf{N}, \\
& \left|L F_{k}\right| \leq L F_{k}^{\max } \quad \forall k \in \mathbf{B} .
\end{aligned}
$$

The generation cost function of the $n$th bus is illustrated as follows [32]:

$$
C_{n}\left(P_{n}^{G}\right)=a_{n}+b_{n} P_{n}^{G}+c_{n}\left(P_{n}^{G}\right)^{2 \$} / h .
$$

\subsection{Power system harmonic model}

The method used in this paper is harmonic injections replacement by current sources with the frequency of the harmonic load [4].
Line impedance in the $h$ th harmonic is expressed as follows:

$$
Z_{h}=R+j h X \text {. }
$$

All loads in the system are represented by resistance parallel to inductors or capacitors given by the following equation:

$$
\begin{gathered}
G_{n}=\frac{P_{n}}{V_{n}^{2}}, \\
Y_{n}=-\frac{Q_{n}}{V_{n}^{2}} .
\end{gathered}
$$

Basic load flow equations can be used to calculate the harmonic voltages of the buses:

$$
\mathbf{I}_{h}=\mathbf{Y}_{h} \times \mathbf{V}_{h}
$$

\subsection{Skin effect}

The skin effect causes the effective resistance of the conductor to increase at higher frequencies where the skin depth is smaller, thus reducing the effective crosssection of the conductor. The skin effect results from opposing eddy currents induced by the varying magnetic field resulting from the alternating current.

The skin effect in a circular conductor was analyzed by Ramo and Whinnery [33]. The skin effect dominates the resistance by increasing frequency. Also, the skin effect on the resistance of a circular conductor is expressed as follows [34]:

$$
\begin{aligned}
\alpha_{R} & =\frac{R_{a c}}{R_{d c}} \\
& =\frac{m r}{2}\left[\frac{\operatorname{ber}(m r) \cdot b e i^{\prime}(m r)-b e i(m r) \cdot b e r^{\prime}(m r)}{\left(b e r^{\prime}(m r)\right)^{2}+\left(b e i^{\prime}(m r)\right)^{2}}\right]
\end{aligned}
$$

$\operatorname{ber}(m r), \operatorname{bei}(m r), b e r^{\prime}(m r), b e i^{\prime}(m r)$ are defined as follows:

$$
\begin{aligned}
& \operatorname{ber}(m r)=1-\frac{(m r)^{4}}{2^{2} \times 4^{2}}+\frac{(m r)^{8}}{2^{2} \times 4^{2} \times 6^{2} \times 8^{2}}-\cdots \\
& \operatorname{bei}(m r)=\frac{(m r)^{4}}{2^{2}}-\frac{(m r)^{6}}{2^{2} \times 4^{2} \times 6^{2}}-\cdots \\
& \operatorname{ber}^{\prime}(m r)=\frac{d}{d(m r)} \operatorname{ber}(m r) \\
& \operatorname{bei}^{\prime}(m r)=\frac{d}{d(m r)} \operatorname{bei}(m r)
\end{aligned}
$$

where $m$ is as below:

$$
m=\sqrt{\omega \mu_{r} \mu_{0} \sigma} .
$$

$\sigma$ is the conductivity of the conductor and is calculated as follows: 
Table 1. Corrections for skin effect in overhead lines [35].

\begin{tabular}{|c|c|c|c|}
\hline Company & Voltage $(\mathbf{k V})$ & Harmonic order & Resistance \\
\hline \multirow[t]{4}{*}{ NGC } & $\begin{array}{l}400,275 \text { (based on } 0.4 \text { sq.in. steel- } \\
\text { core al. conductors) }\end{array}$ & $h \leq 4.21$ & $R_{1}\left(1+\frac{3.45 h^{2}}{192+2.77 h^{2}}\right)$ \\
\hline & 132 & $4.21<h \leq 7.76$ & $R_{1}(0.806+0.105 h)$ \\
\hline & & $h>7.76$ & $R_{1}(0.267+0.485 \sqrt{h})$ \\
\hline & & & $R_{1}\left(1+\frac{0.6465 h^{2}}{192+0.518 h^{2}}\right)$ \\
\hline \multirow[t]{4}{*}{$\mathrm{EDF}$} & 400,225 & $h \leq 4$ & $R_{1}\left(1+\frac{3.45 h^{2}}{192+2.77 h^{2}}\right)$ \\
\hline & & $4<h<8$ & $R_{1}(0.864-0.024 \sqrt{h}+0.105 h)$ \\
\hline & & $h>8$ & $R_{1}(0.267+0.485 \sqrt{h})$ \\
\hline & 150,90 & & $R_{1}\left(1+\frac{0.646 h^{2}}{192+0.518 h^{2}}\right)$ \\
\hline
\end{tabular}

$R_{1}$ is resistance of conductor at power frequency.

$$
\sigma=\frac{1}{\rho}
$$

As an alternative to the above analysis, power companies often use approximations to the skin effect employing correction factors. Typical current corrections utilized by the NGC (UK) and EDF (France) are reported in Table 1 [35].

\section{Proposed algorithm}

Energy pricing has been performed by OPF at the power frequency. Hence, harmonic effects are ignored in the pricing. Harmonics effects, including skin effect, resistance, and reactance elevation as well as occupation of the capacity of the lines should be embedded in OPF calculation and pricing.

\subsection{Lines' capacity occupation}

The occupation of transmission capacity is modeled with a decrease in the transmission capacity via harmonic power flow. The division of the transmission capacity of the lines into fundamental power flow and harmonic power flow is given in Figure 1.

Thus, the transmission capacity of the line for nodal pricing in the presence of harmonics can be written as follows:

$$
L F_{k}^{\max , n e w}=L F_{k}^{\max }-L F_{k, h} .
$$

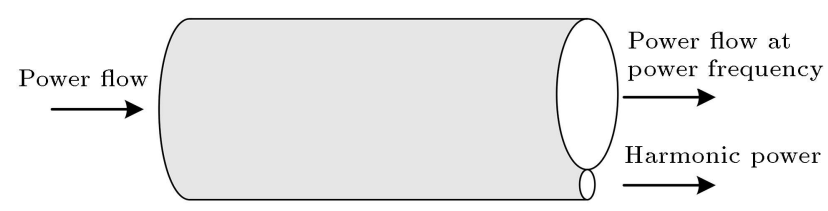

Figure 1. Line's transmission capacity division to fundamental power flow and harmonic power flow.
Harmonic power flowing can be written as follows:

$$
L F_{k, h}=\sum_{h=1} L F_{k}^{h} .
$$

\subsection{Harmonic losses}

Harmonic losses are modeled as additional resistance which is in series with the actual resistance.

Losses caused by total actual and additional resistances in each line in non-harmonic pollution are equal to the losses arising from the actual resistance under the harmonic pollution conditions. Thus, when OPF is performed at the power frequency, harmonic effects have been considered in the calculation:

$$
\begin{aligned}
& \sum_{h=1} R_{k, h} I_{k, h}^{2}=\left(R_{k, 1}+R^{\prime}\right. \\
& \sum_{h=1} R_{k, h} I_{k, h}^{2}=R_{k}^{n e w} I_{1}^{2}, \\
& R_{k}^{\text {new }}=\frac{\sum_{h=1} R_{k, h} I_{k, h}^{2}}{I_{k, 1}^{2}},
\end{aligned}
$$

where $R_{k, h}$ is the $k$ th line resistance at the $h$ th harmonic skin effect obtained from Table 1.

Harmonic power flow facilitates calculating the harmonic current flowing on the $k$ th line from bus $i$ to bus $j$ :

$$
I_{k, h}=\frac{V_{i, h}-V_{j, h}}{Z_{k, h}} .
$$

\subsection{Reactance changing}

Harmonic reactance $\left(X^{h}\right)$ in the $h$ th harmonic is $X^{h}=$ $h X$. Thus, reactances are increased by the harmonic order. Note that the equivalent reactance is calculated 
similar to calculating the equivalent resistance in the presence of harmonic.

The effect of all harmonics on the $k$ th line reactance between bus $i$ and $j$ can be calculated as follows:

$$
Z_{k}=\frac{V_{i}-V_{j}}{I_{k}}
$$

where $I_{k}, V_{i}$, and $V_{j}$ represent the $k$ th line current as well as $i$ th and $j$ th bus voltages, respectively, and can be calculated as follows:

$$
\begin{aligned}
I_{k} & =\sqrt{\sum_{h=1} I_{k, h}^{2}}, \\
V_{i} & =\sqrt{\sum_{h=1} V_{i, h}^{2}} .
\end{aligned}
$$

Then, the equivalent reactance of the $k$ th line can be calculated as:

$$
X_{k}^{n e w}=\sqrt{Z_{k}^{2}-R_{k}^{n e w 2}} .
$$

Figure 2 indicates the flowchart of calculating nodal prices while considering the harmonic effects.

\section{Case study}

\subsection{9-bus system}

A 9-bus system was given in [36]. The characteristics of this system are shown in Tables 2 and 3 , where $R, X$, and $B$ are the resistance, reactance, and susceptance, respectively.

The marginal costs of generators are given by the polynomial model as follows:

$$
\begin{aligned}
& M C_{1}=0.11 P^{2}+5 P+150 \$ / \mathrm{MWh}, \\
& M C_{2}=0.085 P^{2}+1.2 P+600 \$ / \mathrm{MWh}, \\
& M C_{3}=0.1225 P^{2}+P+335 \$ / \mathrm{MWh} .
\end{aligned}
$$

Five cases have been considered for investigating the harmonic effect on local marginal pricing. These cases including harmonic injections are listed in Table 4 .

Nodal prices by considering harmonic effects and LMP deviations are shown in Table 5. LMP deviations are also presented in Figure 3. As can be seen, these deviations have significant values that can result in the generation of inaccurate signals for power markets such as FTR. As can be seen in Table 6, these deviations have occurred while the THD index of currents and voltages have been less than $2.3 \%$ and $1.3 \%$, respectively, and below the limits set by the traditional IEEE519 standard [37].

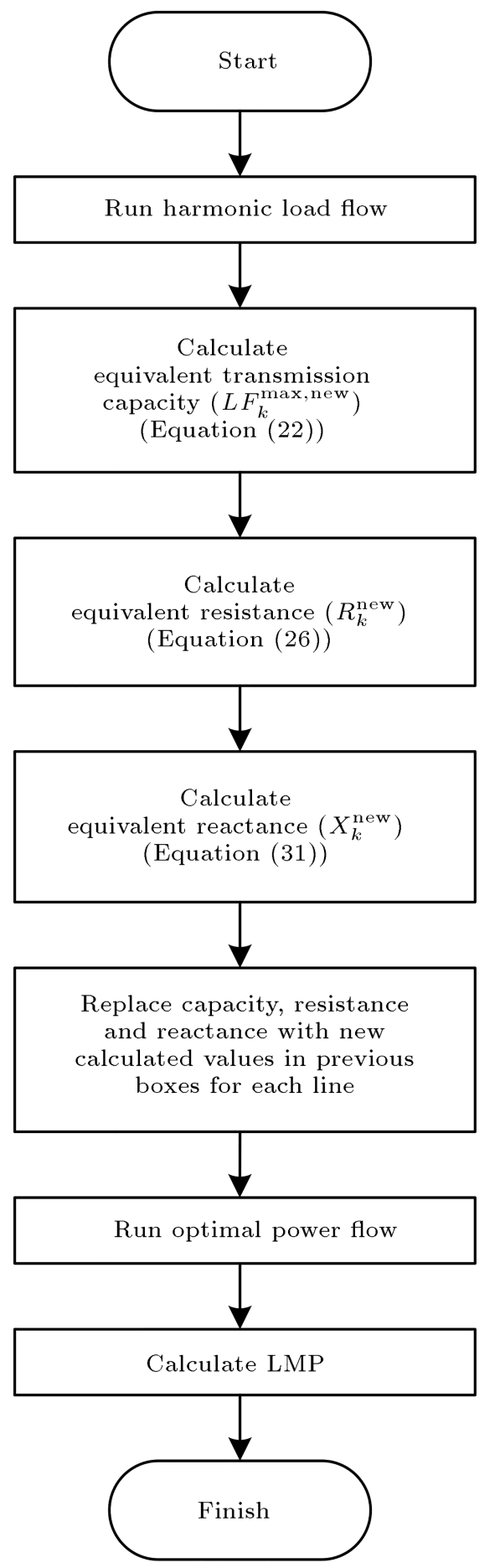

Figure 2. Flowchart of calculating nodal prices by considering harmonic effects.

\subsection{0-bus system}

The 30-bus system given in [38] was tested by harmonic injections, as outlined in Table 7 . The deviation of LMPs due to harmonic effects is illustrated in Figure 4.

As can be seen in Figure 4, the harmonics result in nodal price variations. Nodal prices on both demand and generation side are affected by harmonics. LMP deviations at some busses such as $8,25,26$, and 28 have 
Table 2. Characteristics of lines for the 9-bus system.

\begin{tabular}{ccccccc}
\hline Line & From bus & To bus & $\boldsymbol{R}$ (p.u.) & $\boldsymbol{X}$ (p.u.) & $\boldsymbol{B}$ (p.u.) & MVA rating \\
\hline 1 & 1 & 4 & 0.02 & 0.0576 & 0 & 85 \\
2 & 4 & 5 & 0.017 & 0.092 & 0.158 & 140 \\
3 & 5 & 6 & 0.039 & 0.17 & 0.358 & 80 \\
4 & 3 & 6 & 0.025 & 0.0586 & 0 & 120 \\
5 & 6 & 7 & 0.0119 & 0.1008 & 0.209 & 150 \\
6 & 7 & 8 & 0.0085 & 0.072 & 0.149 & 50 \\
7 & 8 & 2 & 0.018 & 0.0625 & 0 & 180 \\
8 & 8 & 9 & 0.032 & 0.161 & 0.306 & 95 \\
9 & 9 & 4 & 0.01 & 0.085 & 0.176 & 100 \\
\hline
\end{tabular}

Table 3. Characteristics of busses for the 9-bus system.

\begin{tabular}{ccccc}
\hline Bus & Type & $\boldsymbol{P}_{\boldsymbol{D}}(\mathbf{M W})$ & $\boldsymbol{Q}_{\boldsymbol{D}}(\mathbf{M V A r})$ & $\boldsymbol{V}$ (p.u.) \\
\hline 1 & ref & 0 & 0 & 1 \\
2 & PV & 0 & 0 & 1 \\
3 & PV & 0 & 0 & 1 \\
4 & PQ & 0 & 0 & - \\
5 & PQ & 90 & 30 & - \\
6 & PQ & 0 & 0 & - \\
7 & PQ & 100 & 35 & - \\
8 & PQ & 0 & 0 & - \\
9 & PQ & 125 & 50 & - \\
\hline
\end{tabular}

Table 4. Harmonic injections in four cases for the 9-bus system (\%).

\begin{tabular}{|c|c|c|c|c|c|c|c|c|c|c|c|c|}
\hline \multirow{2}{*}{\multicolumn{2}{|c|}{$\begin{array}{c}\text { Case } \\
\text { Harmonic order }\end{array}$}} & \multirow{2}{*}{$\begin{array}{c}\text { Case } 1 \\
3\end{array}$} & \multirow{2}{*}{$\begin{array}{c}\text { Case } 2 \\
3\end{array}$} & \multicolumn{2}{|c|}{ Case 3} & \multicolumn{3}{|c|}{ Case 4} & \multicolumn{4}{|c|}{ Case 5} \\
\hline & & & & 3 & 5 & 3 & 5 & 7 & 3 & 5 & 7 & 11 \\
\hline \multirow{9}{*}{ Bus } & 1 & 0 & 0 & 0 & 0 & 0 & 0 & 0 & 0 & 0 & 0 & 0 \\
\hline & 2 & 0 & 0 & 0 & 0 & 0 & 0 & 0 & 0 & 0 & 0 & 0 \\
\hline & 3 & 0 & 0 & 0 & 0 & 0 & 0 & 0 & 0 & 0 & 0 & 0 \\
\hline & 4 & 0.5 & 1 & 1 & 0 & 1 & 0.5 & 0 & 2 & 0.5 & 0.5 & 0 \\
\hline & 5 & 0.4 & 0.8 & 0 & 0.5 & 2 & 0.8 & 0.2 & 1 & 0.8 & 0 & 0.8 \\
\hline & 6 & 0.8 & 0.8 & 2 & 0.5 & 1.5 & 0 & 1 & 2 & 1.5 & 0 & 0.2 \\
\hline & 7 & 0.6 & 1 & 1.5 & 0.8 & 1.5 & 1 & 0 & 0 & 2 & 1 & 0 \\
\hline & 8 & 0.8 & 0.6 & 0.8 & 0 & 1 & 0.8 & 0.5 & 0.5 & 1.5 & 0.2 & 1 \\
\hline & 9 & 0 & 1 & 1 & 0.8 & 0 & 1 & 1 & 1.5 & 1 & 0.8 & 0 \\
\hline
\end{tabular}

been significant, because of severe congestion occurring at the lines ending at these busses. These significant deviations increase the FTR and the gap between total generation revenue and total consumption cost. It is suggested that consideration of the harmonic effects in pricing and power market enhances the transmission share value.

The contribution of harmonic effects is normally neglected in other OPF calculation methods, while Figure 4 shows that its effects on the calculations may be considerable.

\section{Conclusion}

This paper aimed to investigate the harmonic effects on nodal prices. The resistance, reactance, and transmission capacities for each line were replaced for calculating Locational Marginal Pricing (LMP) with harmonics.

The results of simulations revealed the effects of harmonics on LMPs and changes in the nodal price values on the generation and demand side of the power market, while harmonics were below the standard level. 
Table 5. New nodal prices by considering harmonic effects.

\begin{tabular}{|c|c|c|c|c|c|c|c|c|c|c|}
\hline & Bus & 1 & 2 & 3 & 4 & 5 & 6 & 7 & 8 & 9 \\
\hline & LMP (\$/MWh) & 23.6121 & 22.0659 & 29.9089 & 28.1364 & 30.0252 & 31.5721 & 33.7358 & 22.9207 & 26.9443 \\
\hline \multirow{2}{*}{ Case 1} & LMP' (\$/MWh) & 23.5640 & 22.1211 & 29.8733 & 28.6808 & 29.7986 & 31.4865 & 34.0925 & 22.9647 & 26.8707 \\
\hline & Deviation $(\$ / M W h)$ & -0.0481 & 0.0552 & -0.0356 & 0.5443 & -0.2266 & -0.0855 & 0.3568 & 0.0440 & -0.0736 \\
\hline \multirow{2}{*}{ Case 2} & LMP' (\$/MWh) & 23.5642 & 22.1171 & 29.8789 & 28.6813 & 29.8034 & 31.4928 & 34.1010 & 22.9604 & 26.8689 \\
\hline & Deviation $(\$ / M W h)$ & -0.0479 & 0.0513 & -0.0299 & 0.5449 & -0.2218 & -0.0793 & 0.3653 & 0.0398 & -0.0754 \\
\hline \multirow{2}{*}{ Case 3} & LMP' $^{\prime}(\$ / \mathrm{MWh})$ & 23.5642 & 22.1083 & 29.8921 & 28.6828 & 29.8167 & 31.5074 & 34.1216 & 22.9509 & 26.8647 \\
\hline & Deviation $(\$ / M W h)$ & -0.0479 & 0.0424 & -0.0167 & 0.5464 & -0.2085 & -0.0646 & 0.3858 & 0.0302 & -0.0795 \\
\hline \multirow{2}{*}{ Case 4} & LMP' (\$/MWh) & 23.5651 & 22.1099 & 29.8888 & 28.6701 & 29.8072 & 31.5037 & 34.1168 & 22.9527 & 26.8602 \\
\hline & Deviation $(\$ / M W h)$ & -0.0470 & 0.0441 & -0.0201 & 0.5337 & -0.2180 & -0.0684 & 0.3810 & 0.0320 & -0.0841 \\
\hline \multirow{2}{*}{ Case 5} & LMP' $^{\prime}(\$ / \mathrm{MWh})$ & 23.5648 & 22.0877 & 29.9220 & 28.6833 & 29.8396 & 31.5405 & 34.1627 & 22.9288 & 26.8564 \\
\hline & Deviation $(\$ / M W h)$ & -0.0472 & 0.0219 & 0.0132 & 0.5469 & -0.1856 & -0.0316 & 0.4270 & 0.0081 & -0.0878 \\
\hline
\end{tabular}

Table 6. THD index of currents and voltages.

\begin{tabular}{rcccccccccc}
\hline Case & \multicolumn{2}{c}{ Case 1 } & \multicolumn{2}{c}{ Case 2 } & \multicolumn{2}{c}{ Case 3 } & \multicolumn{2}{c}{ Case 4 } & \multicolumn{2}{c}{ Case 5 } \\
\hline THD & THD(i) & THD(v) & THD(i) & THD(v) & THD(i) & THD(v) & THD(i) & THD(v) & THD(i) & THD(v) \\
\hline 1 & 0.000 & 0.369 & 0.000 & 0.805 & 0.000 & 0.999 & 0.000 & 1.260 & 0.000 & 1.265 \\
2 & 0.000 & 0.303 & 0.000 & 0.800 & 0.000 & 0.888 & 0.000 & 1.127 & 0.000 & 1.456 \\
3 & 0.000 & 0.278 & 0.000 & 0.807 & 0.000 & 0.882 & 0.000 & 1.104 & 0.000 & 1.276 \\
4 & 0.500 & 0.376 & 1.000 & 0.820 & 1.000 & 1.017 & 1.118 & 1.284 & 2.121 & 1.289 \\
Bus 5 & 0.400 & 0.350 & 0.800 & 0.885 & 0.500 & 1.195 & 2.163 & 1.243 & 1.510 & 1.587 \\
6 & 0.800 & 0.284 & 0.800 & 0.826 & 2.062 & 0.903 & 1.803 & 1.130 & 2.508 & 1.306 \\
7 & 0.600 & 0.294 & 1.000 & 0.832 & 1.700 & 0.905 & 1.803 & 1.156 & 2.236 & 1.644 \\
8 & 0.800 & 0.307 & 0.600 & 0.812 & 0.800 & 0.901 & 1.375 & 1.144 & 1.881 & 1.478 \\
9 & 0.000 & 0.419 & 1.000 & 0.798 & 1.281 & 0.933 & 1.414 & 1.771 & 1.972 & 1.445 \\
\hline
\end{tabular}

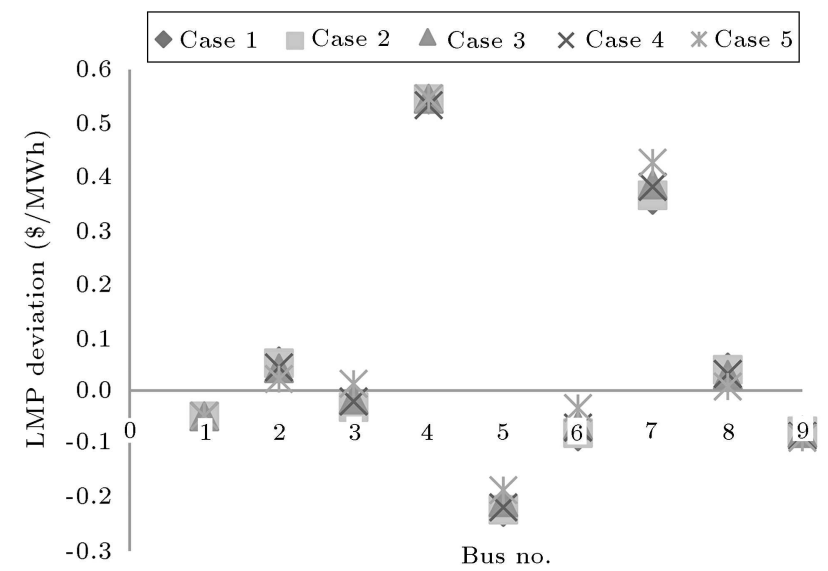

Figure 3. LMP deviation in the 9-bus system.

These deviations led to Financial Transmission Right (FTR) alterations. The elevation of the total cost was another effect of harmonics on the power systems.

Energy pricing was performed using optimal

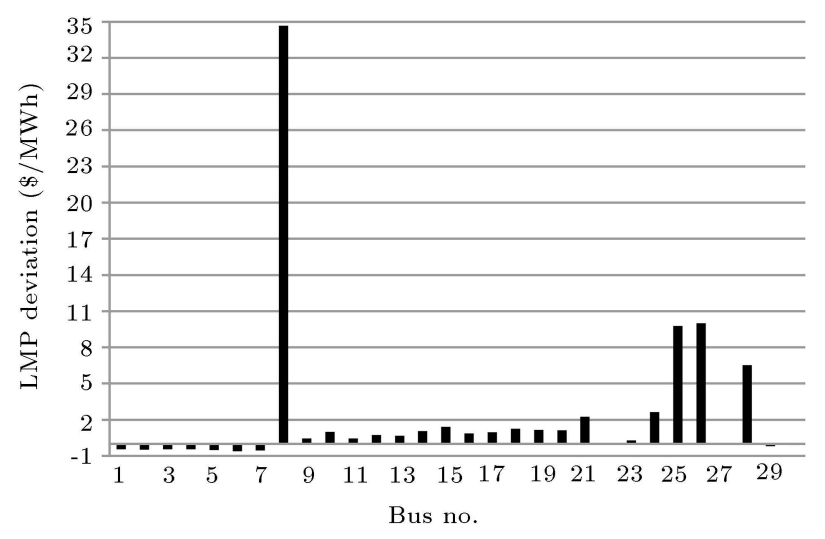

Figure 4. LMP deviation in the 30-bus system.

power flow at power frequency, while harmonic effects were ignored in pricing. Numerical results exhibited the difference between Optimal Power Flow (OPF) results with and without consideration of harmonics. Consequently, capturing harmonics in the pricing pro- 
Table 7. Harmonic injections for the 30-bus system (\%).

\begin{tabular}{|c|c|c|c|c|}
\hline Bus & 3rd harmonic & 5th harmonic & 7th harmonic & 11th harmonic \\
\hline 1 & 0 & 0 & 0 & 0 \\
\hline 2 & 0 & 0 & 0 & 0 \\
\hline 3 & 1 & 0 & 0 & 0 \\
\hline 4 & 1.5 & 2 & 0 & 0 \\
\hline 5 & 1 & 0 & 0 & 0 \\
\hline 6 & 0 & 0 & 0 & 0 \\
\hline 7 & 0 & 0 & 2 & 0 \\
\hline 8 & 0 & 1 & 0 & 0 \\
\hline 9 & 0 & 1.5 & 0 & 1.7 \\
\hline 10 & 0 & 0 & 0 & 0 \\
\hline 11 & 0 & 0 & 1.5 & 0 \\
\hline 12 & 2 & 0 & 0 & 0 \\
\hline 13 & 0 & 0 & 0 & 0 \\
\hline 14 & 0 & 1.7 & 0 & 1.2 \\
\hline 15 & 0 & 0 & 0 & 0 \\
\hline 16 & 0.8 & 0 & 0 & 0 \\
\hline 17 & 1 & 0 & 1.5 & 0 \\
\hline 18 & 0 & 0 & 0 & 1 \\
\hline 19 & 0 & 0.8 & 0 & 0 \\
\hline 20 & 0 & 0 & 0 & 0 \\
\hline 21 & 0 & 0 & 0 & 0 \\
\hline 22 & 0 & 0 & 2 & 0 \\
\hline 23 & 1.7 & 0 & 0 & 1.5 \\
\hline 24 & 0 & 0 & 0 & 0 \\
\hline 25 & 0 & 2 & 0 & 0 \\
\hline 26 & 0 & 0 & 0 & 0 \\
\hline 27 & 0 & 0 & 1 & 0 \\
\hline 28 & 2 & 0 & 0 & 2 \\
\hline 29 & 0 & 1 & 0 & 0 \\
\hline 30 & 0 & 0 & 0 & 0 \\
\hline
\end{tabular}

cess helps the power market send and receive more accurate signals and enhance the accuracy of power market signals, which can culminate in better decisionmaking.

\section{Nomenclature}

$\lambda_{r e f} \quad$ The price at the slack bus

$\lambda_{\text {cong }} \quad$ Marginal congestion price

$\lambda_{\text {loss }} \quad$ Marginal congestion price

$\mathbf{N} \quad$ Set of buses

B Set of branches

G Controllable generators located at a subset $G \subseteq N$ of the system buses

$C_{n}\left(P_{n}^{G}\right)$
$P_{n}^{G}$

$Q_{n}^{G}$

$V_{n}$

$\delta_{n}$

$L F_{k}$

$L F_{k}^{\max }$

$a_{n}, b_{n}, c_{n}$

$P_{n}^{G}$

$H$

$R$

$X$

$G_{n}$

$Y_{n}$
Injected active power at bus $n$

Injected reactive power at bus $n$

The $n$th bus voltage

The $n$th bus voltage angle

The $k$ th line flow

The $k$ th line congestion

Fuel cost coefficients

Injected active power at bus $n$

Harmonic order

Line resistance at power frequency

Line reactance at power frequency Conductivity of load at the $n$th bus Admittance of load at the $n$th bus 
$\mathbf{I}_{h}$

$\mathbf{V}_{h}$

$\mathbf{Y}_{h}$

$R_{d c} \quad$ Resistance of conductor when current distribution is uniform

$R_{a c} \quad$ Resistance of conductor when current distribution is alternating sinusoidal

ber(.) Real part of the Bessel function of the first kind and zero order

bei(.) Imaginary part of the Bessel function of the first kind and zero order

ber'(.) Derivative of $\operatorname{ber}($.

bei' ${ }^{\prime}$.) Derivative of bei( . )

$r \quad$ Conductor radius

$\sigma \quad$ The conductivity of the conductor

$\rho \quad$ The resistivity of the conductor

$L F_{k}^{\max , n e w}$ Equivalent $k$ th line transmission capacity in the presence of harmonic

$L F_{k}^{\max } \quad k$ th line transmission capacity at power frequency

$L F_{k, h} \quad$ Harmonic power flowing in $k$ th line

$L F_{k}^{h} \quad h$ th harmonic power flowing in the $k$ th line

$R_{k, h} \quad k$ th line resistance at the $h$ th harmonic affected skin effect

$R_{k n, 1} \quad k$ th line resistance at power frequency

$R_{k}^{\prime} \quad$ Additional resistance series with actual resistance for the $k$ th line

$R_{k}^{\text {new }} \quad$ Equivalent resistance in presence of harmonic for the $k$ th line

$I_{k, h} \quad k$ th line current at the $h$ th harmonic frequency

$I_{k, 1} \quad k$ th line current at power frequency

$Z_{k, h} \quad k$ th line impedance at the $h$ th harmonic frequency

$V_{i, h} \quad i$ th bus voltage at the $h$ th harmonic frequency

$Z_{k} \quad k$ th line impedance

$I_{k} \quad k$ th line current

$V_{i} \quad i$ th bus voltages

\section{References}

1. Schweppe, F.C., Caramanis, M.C., Tabors, R.D., et al., Spot Pricing of Electricity, Springer US (1988).

2. Yang, Z., Bose, A., Zhong, H., et al., "LMP revisited: A linear model for the loss-embedded LMP", IEEE Trans. Power Syst., 32(5), pp. 4080-4090 (2017).
3. Leite da Silva, A.M. and de Carvalho Costa, J.G. "Transmission loss allocation: part I-single energy market", IEEE Trans. Power Syst., 18(4), pp. 13891394 (2003).

4. Norouzi, H., Abedi, S., Jamalzadeh, R., et al. "Modeling and investigation of harmonic losses in optimal power flow and power system locational marginal pricing", Energy, 68, pp. 140-147 (2014).

5. Vaishya, S.R. and Sarkar, V. "Implementation of lossy FTRs for perfect risk hedging under the marginal loss pricing", IET Gener. Transm. Distrib., 11(1), pp. 166173 (2017).

6. Vaishya, S.R. and Sarkar, V. "Designing option FTRs for the lossy FTR system", IET Gener. Transm. Distrib., 12(9), pp. 2132-2139 (2018).

7. Avinash, D. and Chalapathi, B. "MW-Mile method considering the cost of loss allocation for transmission pricing", 2015 Conf. Power, Control. Commun. Comput. Technol. Sustain. Growth, IEEE, pp. 128-131 (2015).

8. Mackay, L., Ramirez-Elizondo, L., and Bauer, P. "Current pricing: Avoiding marginal losses in locational marginal prices for DC grids", 2017 IEEE Manchester PowerTech, IEEE, pp. 1-6 (2017).

9. Litvinov, E., Zheng, T., Rosenwald, G., et al. "Marginal loss modeling in LMP calculation", IEEE Trans. Power Syst., 19(2), pp. 880-888 (2004).

10. Shaloudegi, K., Madinehi, N., Hosseinian, S.H., et al. "A novel policy for locational marginal price calculation in distribution systems based on loss reduction allocation using game theory", IEEE Trans. Power Syst., 27(2), pp. 811-820 (2012).

11. O'Connell, N., Wu, Q., фstergaard, J., et al. "Dayahead tariffs for the alleviation of distribution grid congestion from electric vehicles", Electr. Power Syst. Res., 92, pp. 106-114 (2012).

12. Li, R., Wu, Q., and Oren, S.S. "Distribution locational marginal pricing for optimal electric vehicle charging management", IEEE Trans. Power Syst., 29(1), pp. 203-211 (2014).

13. Huang, S., Wu, Q., Oren, S.S., et al. "Distribution locational marginal pricing through quadratic programming for congestion management in distribution networks", IEEE Trans. Power Syst., 30(4), pp. 21702178 (2015).

14. Hamoud, G. and Bradley, I. "Assessment of transmission congestion cost and locational marginal pricing in a competitive electricity market", IEEE Trans. Power Syst., 19(2), pp. 769-775 (2004).

15. Sun, J. and Lo, K.L. "A congestion management method with demand elasticity and PTDF approach", 2012 47th Int. Univ. Power Eng. Conf., IEEE, pp. 1-6 (2012).

16. Talacek, P.J. and Watson, N.R. "Marginal pricing of harmonic injections: an analysis of the resulting payments", IEEE Trans. Power Syst., 17(3), pp. 640645 (2002). 
17. Aiello, M., Cataliotti, A., Favuzza, S., et al. "Theoretical and experimental comparison of total harmonic distortion factors for the evaluation of harmonic and interharmonic pollution of grid-connected photovoltaic systems", IEEE Trans. Power Deliv., 21(3), pp. 13901397 (2006).

18. Kim, K., Suk Song, C., Byeon, G., et al. "Power demand and total harmonic distortion analysis for an EV charging station concept utilizing a battery energy storage system", J. Electr. Eng. Technol., 8(5), pp. 1234-1242 (2013).

19. Hojabri, M. and Toudeshki, A. "Power quality consideration for off-grid renewable energy systems", Energy Power Eng., 5(5), pp. 377-383 (2013).

20. Marini, A., Ghazizadeh, M.S., Mortazavi, S.S., et al. "A harmonic power market framework for compensation management of DER based active power filters in microgrids", Int. J. Electr. Power Energy Syst., 113, pp. 916-931 (2019).

21. Narimani, M., Hosseinian, S.H., and Vahidi, B. "A method for harmonic power tracing by using upstream and downstream distribution matrices", Electr. Power Components Syst., 47(13), pp. 1169-1179 (2019).

22. Li, C.S., Bai, Z.X., Xiao, X.Y., et al. "Research of harmonic distortion power for harmonic source detection", 2016 17th Int. Conf. Harmon. Qual. Power, IEEE, pp. 126-129 (2016).

23. Wu, T., Ping Shum, P., Sun, Y., et al. "Third harmonic generation with the effect of nonlinear loss", J. Light. Technol., 34(4), pp. 1274-1280 (2016).

24. Kumar, D. and Zare, F. "Harmonic analysis of grid connected power electronic systems in low voltage distribution networks", IEEE J. Emerg. Sel. Top. Power Electron., 4(1), pp. 70-79 (2016).

25. Mesas, J.J., Sainz, L., and Sala, P. "Statistical study of personal computer cluster harmonic currents from experimental measurements", Electr. Power Components Syst., 43(1), pp. 56-68 (2015).

26. Bharatwaj, V.N. and Abhyankar, A.R. "Max-min fair financial transmission rights payment-based AC optimal power flow locational marginal price decomposition", IET Gener. Transm. Distrib., 8(10), pp. 17241732 (2014).

27. Sarkar, V. and Khaparde, S.A. "Optimal LMP decomposition for the ACOPF calculation", IEEE Trans. Power Syst., 26(3), pp. 1714-1723 (2011).

28. http://www.caiso.com/23cf/23cfe2c91d880.pdf.

29. Monica Gokul, C.H. and Sarada, K. "AC optimal power flow calculation for locational marginal pricing", Indian J. Sci. Technol, 8(17), pp. 1-6 (2015).

30. Carpentie, J. "Contribution a l'étude du dispatching économique", Bull. la Société Française des Electr., 3, pp. 431-447 (1962).
31. Dommel, H. and Tinney, W. "Optimal power flow solutions", IEEE Trans. Power Appar. Syst., PAS87(10), pp. 1866-1876 (1968).

32. Yang, Z., Zhong, H., Xia, Q., et al. "Solving OPF using linear approximations: Fundamental analysis and numerical demonstration", IET Gener. Transm. Distrib., 11(17), pp. 4115-4125 (2017).

33. Ramo, S. and Whinnery, J.R., Fields and Waves in Modern Radio, Second Ed., John Wiley \& Sons, Ltd, New York (1962).

34. Mahmoud, A. and Shultz, R. "A method for analyzing harmonic distribution in A.C. power systems", IEEE Trans. Power Appar. Syst., PAS-101(6), pp. 18151824 (1982).

35. Arrillaga, J. and Watson, N.R., Power System Harmonics, John Wiley \& Sons, Ltd, Chichester, UK (2003).

36. Chow, J.H., Kokotovicć, P.V., and Thomas, R.J., Systems and Control Theory for Power Systems, SpringerVerlag (1995).

37. "519-2014-IEEE recommended practice and requirements for harmonic control in electric power systems", https://standards.iee.org/standard/519-201 4.html.

38. Alsac, O. and Stott, B. "Optimal load flow with steady-state security", IEEE Trans. Power Appar. Syst., PAS-93(3), pp. 745-751 (1974).

\section{Biographies}

Mahmood Narimani was born in Iran. He received $\mathrm{BS}$ and MS degrees in Electrical Engineering from Amirkabir University of Technology (AUT), Tehran, Iran in 2011 and 2013. Presently, he is a PhD student at the Department of Electrical Engineering of Amirkabir University of Technology, Tehran, Iran. His main fields of research are power quality, power market, restructuring, and deregulation in power systems.

Seyed Hossein Hosseinian was born in Iran. He received the $\mathrm{PhD}$ degree from the Electrical Engineering Department, University of Newcastle, Newcastle upon Tyne, U.K. in 1995. At the present, he is a Professor at the Electrical Engineering Department at Amirkabir University of Technology (AUT), Tehran, Iran. He is the author of four books in the field of power systems. He is also the author and co-author of over 200 technical papers. His special fields of interest include transient in power systems, power quality, restructuring, and deregulation in power systems. 